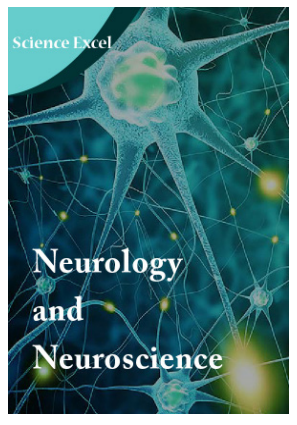

\section{*Correspondence}

\section{Dimitar Maslarov}

Neurology Clinic, University First MHAT "St. John Krastitel" - Sofia, Bulgaria,

Tel: 35929152140

E-mail: maslarovdb@abv.bg

- Received Date: 04 May 2020;

- Accepted Date: 22 May 2020;

- Publication Date: 25 May 2020.

\section{Keywords}

ischemic stroke, transient ischemic attack, Nattokinase, prevention

\section{Copyright}

(c) 2020 Science Excel. This is an openaccess article distributed under the terms of the Creative Commons Attribution 4.0 International license.

\title{
Use of Nattokinase in patients with ischemic stroke and transient ischemic attacks
}

\author{
Dimitar Maslarov ${ }^{1,2^{*}}$ and Desislava Drenska ${ }^{1}$ \\ 'Neurology Clinic, University First MHAT "St. John Krastitel" - Sofia, Bulgaria \\ ${ }^{2}$ Medical College "Y. Filaretova", Medical University of Sofia, Bulgaria
}

\begin{abstract}
The concept of Nattokinase as an alternative to the standard prevention in acute ischemic cerebrovascular incidents is interesting and promising.

Two groups of patients were considered - ones taking Nattokinase at a dose of $160 \mathrm{mg}, 3000$ fibrinolytic units of activity for 24 hours $(n=96)$ and a control group, in which the secondary prevention was based on antithrombotic drugs - Acetylsalicylic acid (325/100 mg) and/or Clopidogrel (75 mg) daily ( $n=95)$.

At a 6-month follow-up interval, no statistically significant difference was observed in the vascular (brain and cardiac) complications between the two categories monitored. The occurrence of adverse reactions in both groups was also statistically insignificant, in support of the reported literature and own initial data.
\end{abstract}

\section{Introduction}

Antithrombotic drugs (Class I, Level of Evidence A) are used for secondary prevention in patients with cerebral infarction and transient ischemic attacks (TIA) [1]. It is essential to reduce the risk of subsequent events following acute ischemic cerebrovascular events [2].

The antiplatelet action of Acetylsalicylic acid (ASA) does not occur in all patients and between $8 \%$ and $45 \%$ of people are „aspirin resistant” [3]. Clopidogrel is more effective than self-administered ASA and is globally recommended for secondary prevention of cerebral infarction [4,5]. Between $5 \%$ and $30 \%$ of patients receiving Clopidogrel show low or lack of efficacy, which is known as the term „clopidogrel resistance” $[6,7]$.

In a study of 1186 patients affected by ischemic stroke that have been monitored within 3 years [8], 432 of them have received ASA as a secondary prevention treatment, and 382 - Clopidogrel. In the group with ASA administration, 32 patients underwent secondary and 4 - third hospital admission. Of these, 25 had a new cerebral infarction and $3 \mathrm{had}$ an intracerebral hematoma. Among the patients undergoing secondary prevention treatment with Clopidogrel there were 33 re-admissions and 3 - for the third time, as 26 were for a new ischemic stroke and 1 for parenchymal intracerebral haemorrhage.

The incidence of recurrent cerebrovascular accidents, including in the self-cited observation demonstrates complete or partial clinical resistance to ASA and/or Clopidogrel (in some cases), regardless of the proven efficiency of these drugs. This directs research interest in the discovery and development of new active molecules and combinations, aiming successful secondary prevention in each patient with cerebral infarction and TIAs in daily practice.

According to numerous publications the enzyme Nattokinase has a neuroprotective mechanism of action (as a result of proteolytic, anti-inflammatory and anti-apoptotic effects) in experimental models of ischemic stroke and slows the development of atherosclerosis [9-11].

Nattokinase is an alkaline protease of 257 amino acid parts with multilayer properties, derived from fermented soy product (Natto), a traditional food in Asia $[12,13]$. Based on in vitro and in vivo studies, its specific characteristics have been identified potential fibrinolytic and antithrombotic activity in arterial and venous thrombosis, decrease in blood viscosity and erythrocyte aggregation and a positive effect on controllable risk factors such as arterial hypertension and dyslipidemia [14-16].

In $2012 \mathrm{~J}$-M Wang et al. reported that in animals with experimentally induced focal cerebral ischemia Nattokinase reduced fibrinogen levels and reduced infarction volume by $54-68 \%$. These processes were performed in a cascade-mediated pathway similar to ASA $[9,17]$.

Based on an established model of photothrombotic cerebral infarction in mice Y-J. Ahn et al. [18], confirm the neuroprotective effect of Nattokinase by reducing arterial thrombosis and stroke volume at the 4-th hour of therapy $(26.2 \pm$ $\left.6.5 \mathrm{~mm}^{3}\right)$ compared to controls $\left(42.9 \pm 2.5 \mathrm{~mm}^{3}\right)$. In addition, there is an improvement noted in blood flow in the ischemic area of injury and in the nonischemic area [9].

We present results for the use of oral Nattokinase as an alternative for secondary prevention in acute ischemic cerebrovascular accidents. 

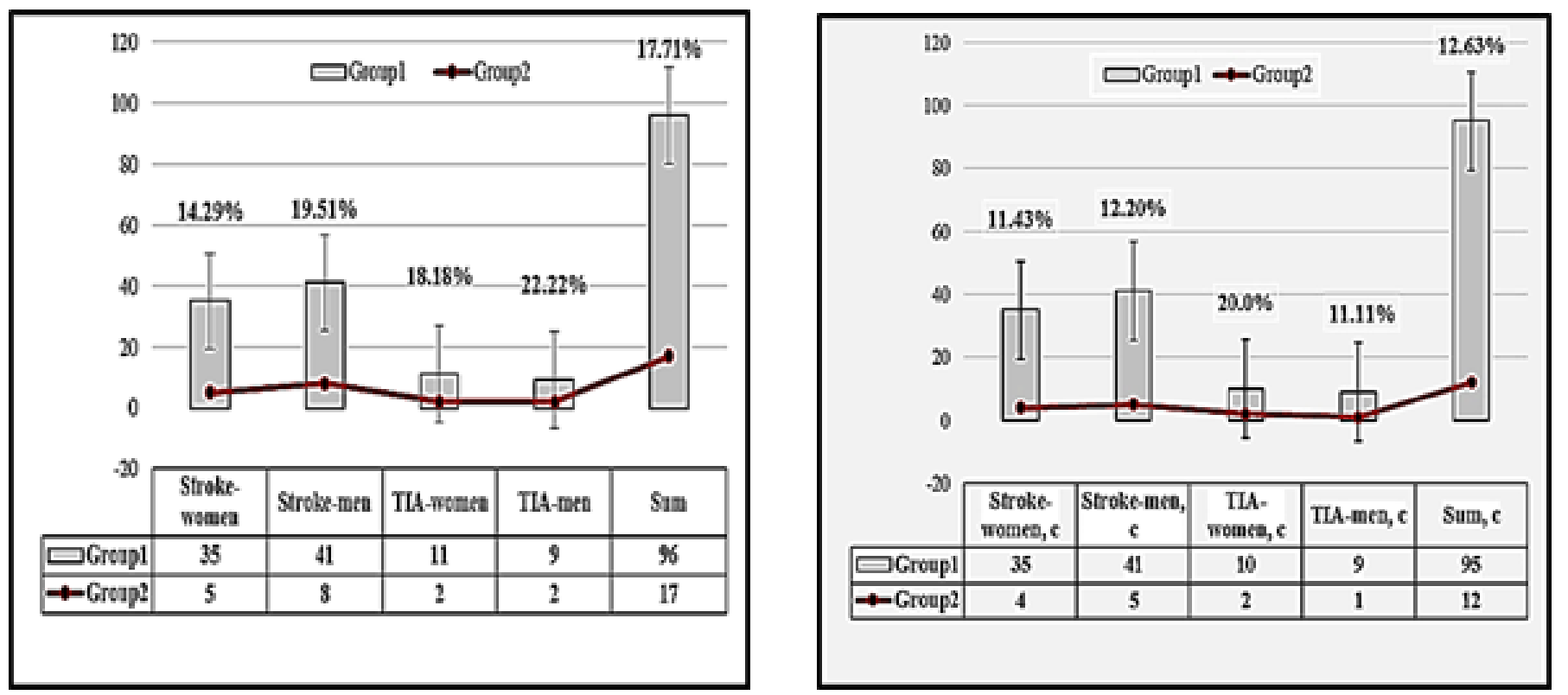

Figure 1. Frequency distribution of accompanying malignant disease in patients with stroke and TIA receiving Nattokinase (1.1) and in controls (1.2).

*With "Group 1" conditionally denoted the studied subjects and with "Group 2" - concomitant oncological comorbidity.

\section{Model and methods}

During the period 01.2017-03.2019 96 patients with ischemic stroke and TIA who underwent treatment at the Clinic of Neurology, University First MHAT - Sofia started taking Nattokinase at a dose of $160 \mathrm{mg}, 3000$ fibrinolytic units for 24 hours once daily. A standard diagnostic and therapeutic panel according to the national Guidelines and Recommendations were performed as well as follow-up meetings. Patients and caregivers have been informed that this is a product with the official registration as a food supplement $\left(\right.$ Nataspin $\mathrm{H}^{\circ}$ ), and an informed consent for clinical follow-up was obtained for each participant and/or companion.

For the purpose of the comparative analysis, the trial included a control group of 95 other patients with stroke and TIA in which secondary prevention was based on antithrombotic drugs - ASA (325/100mg) and/or Clopidogrel (75mg) daily. The characteristics of the patients monitored (taking Nattokinase and control group) are presented in Table 1.

\begin{tabular}{|l|c|c|}
\hline Clinical characteristics & $\begin{array}{c}\text { Patients receiving } \\
\text { Nattokinase, } \mathrm{n}=96\end{array}$ & Control group, $\mathrm{n}=95$ \\
\hline Age \pm SD & $74.6 \pm 7.5$ & $71.9 \pm 8.2$ \\
\hline Sex & & \\
Female & $46(47.9 \%)$ & $45(47.4 \%)$ \\
Male & $50(52.1 \%)$ & $50(52.6 \%)$ \\
\hline With ischemic stroke & $76(79.2 \%)$ & $76(80.0 \%)$ \\
Female & $35(36.5 \%)$ & $35(36.8 \%)$ \\
Males & $41(42.7 \%)$ & $41(43.2 \%)$ \\
\hline With TIA & $20(20.8 \%)$ & $19(20.0 \%)$ \\
Female & $11(11.4 \%)$ & $10(10.5 \%)$ \\
Males & $9(9.4 \%)$ & $9(9.5 \%)$ \\
\hline
\end{tabular}

Table 1. Baseline characteristics of the study participants.
The main indications for selection are: concomitant gastrointestinal disorders associated with a moderate and high risk of bleeding (62.5\%), history of cerebral hemorrhage (6.3\%), rare episodes of atrial fibrillation/flutter (12.5\%), individual intolerance or unwillingness for treatment with antithrombotic drugs (18.7\%). In $17.7 \%$ of patients there was comorbidity with cancer compared to $12.6 \%$ of the control group (Figure 1).

\section{Results and discussion}

The Trial of ORG 10172 in Acute Stroke Treatment (TOAST) is conditionally used in this statement, according to which ischemic cerebrovascular accidents can be divided in 5 groups [19] with atherosclerosis of large blood vessels - aorta, carotid and vertebral arteries cardioembolic with engaging of the small blood vessels other subtypes with determined etiology (due to migraine, vasculitis, vasculopathies, venous thrombosis) cryptogenic - with unknown etiology.

According to TOAST in the group of patients with cerebral infarction and TIA, who were treated with Nattokinase $(n=96)$ the main etiologic factors are athero- and arteriolosclerosis - in $87.5 \%$ of all cases, and only $12.5 \%$ of them can be considered as caused by cardiac sources of embolism. In the control group $(n=95)$ the leading causes are large-artery atherosclerosis and small vessel occlusion - in $92.6 \%$ versus $7.4 \%$ of cases with possible cardioembolic etiology

The analysis includes patients with ischemic strokes and TIA (Nattokinase group and a control group) with predominantly thrombotic origin.

The thesis of our research paper is the application of Nataspin $\mathrm{H}^{\bullet}$ as a mean of secondary prevention in two directions - effectiveness and safety. Cerebrovascular accidents with mixed or cryptogenic etiology are not included in the representative samples.

Patients' response was monitored at 1, 3 and 6-month intervals. In both groups around or under $8.0 \%$ of subjects did not attend a scheduled follow-up visit.

In patients with Nattokinase for a period of 30 days, one case of TIA with criteria for previous high-risk profile, and two cases of ischemic stroke were identified, one of them with severe neurological deficit. 


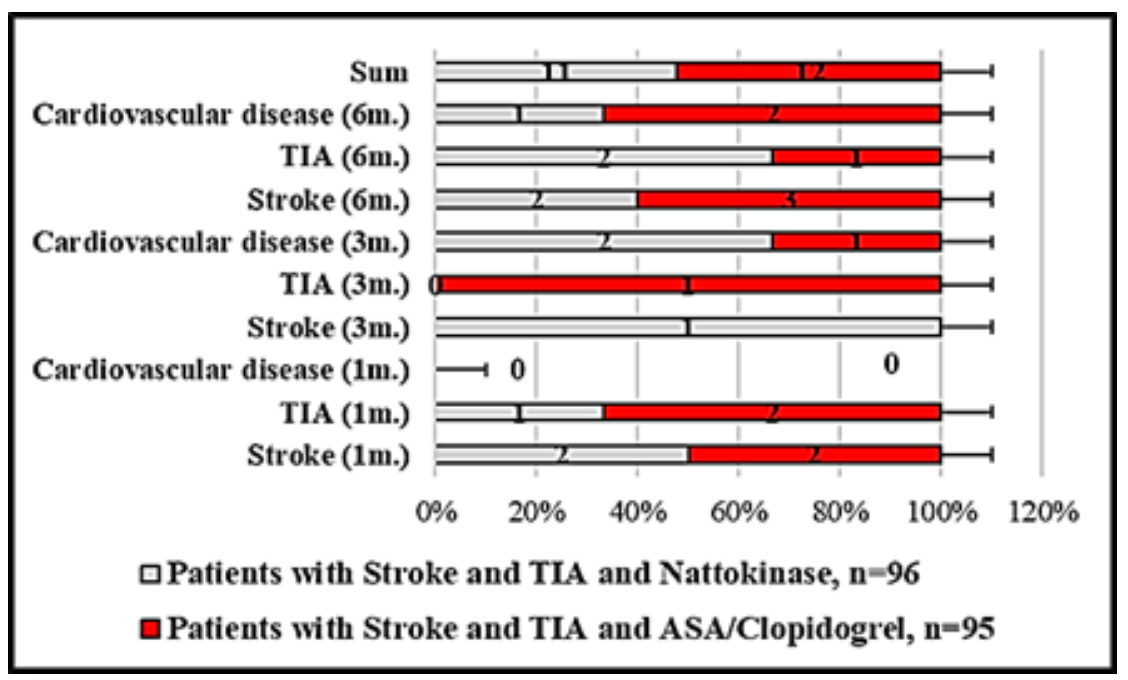

Figure 2. Vascular (cerebral and cardiac) incidents occurred within the 1,3- and 6-month follow-up period between the two groups.

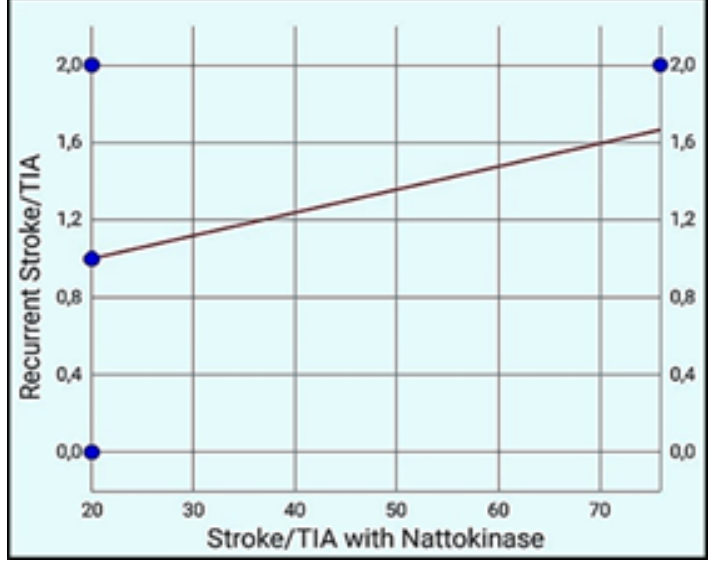

Figure 3.1

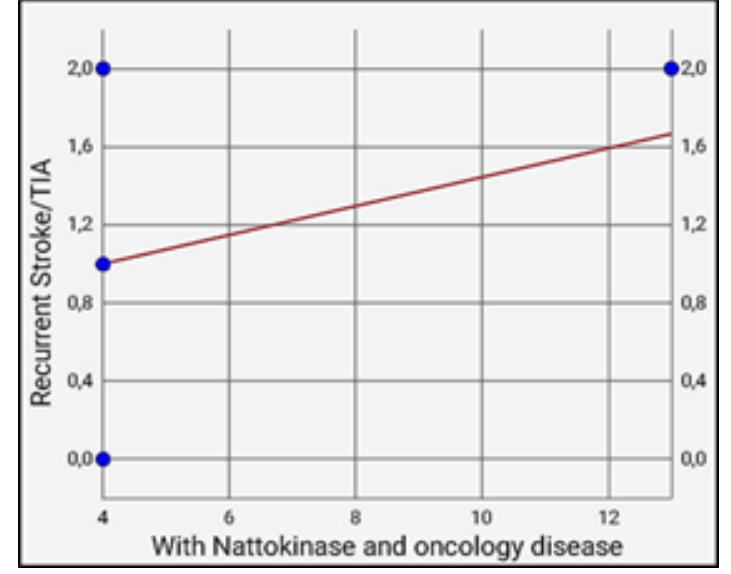

Figure 3.2

Figure 3. At 1,3 and 6 month intervals - recurrent Stroke/TIA $=0.0119$ (Stroke/TIA with Nattokinase) $+0.7619 . R=0.4472, R^{2}=0.2, P=$ 0.3739 (Figure 3.1) and recurrent Stroke/TIA $=0.0741$ (Stroke/TIA with cancer comorbidity and Nattokinase) $+0.7037 . R=0.4472, R^{2}=0.2, P$ $=0.3739$ (Figure 3.2).

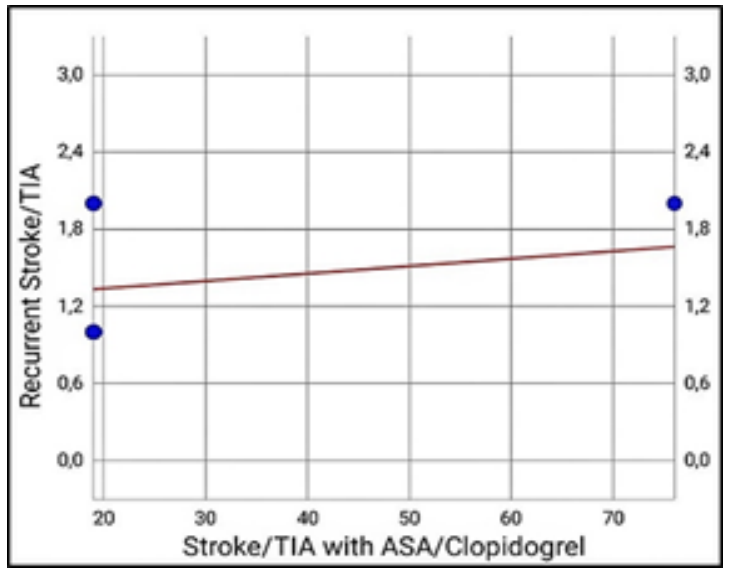

Figure 4.1

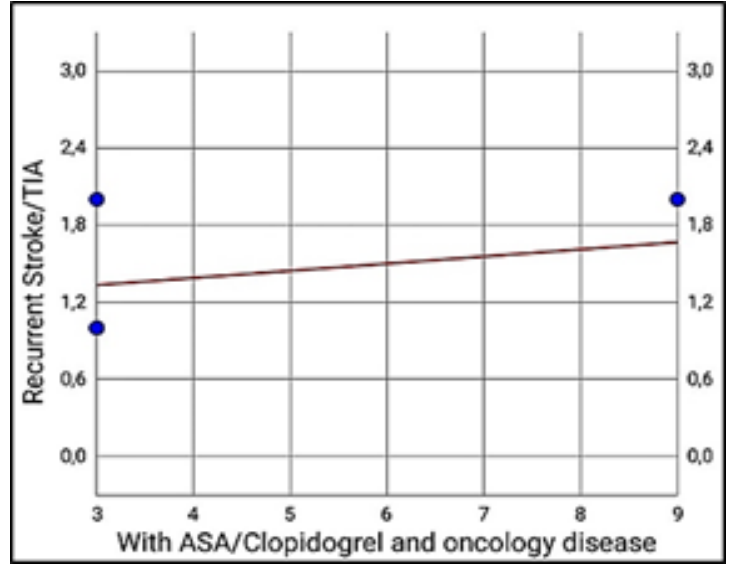

Figure 4.2

Figure 4. At 1, 3 and 6 month intervals - recurrent Stroke/TIA $=0.0058$ (Stroke/TIA with ASA/Clopidogrel) $+1.2222 . R=0.1741, \mathrm{R}^{2}=0.0303$, $\mathrm{P}=0.7415$ (Figure 4.1) and recurrent Stroke/TIA $=0.0556$ (Stroke/TIA with cancer comorbidity and ASA/Clopidogrel) $+1.1667 . \mathrm{R}=0.1741, \mathrm{R}^{2}$ $=0.0303, \mathrm{P}=0.7415$ (Figure 4.2). 


\begin{tabular}{|l|c|c|c|}
\hline & Recurrent Stroke/TIA & Stroke/TIA with Nattokinase & $\begin{array}{c}\text { Stroke/TIA with oncological } \\
\text { comorbidity and Nattokinase }\end{array}$ \\
\hline Recurrent Stroke/TIA & ---- & $0.0011^{*}$ & 0.7718 \\
\hline Stroke/TIA with Nattokinase & $0.0011^{*}$ & ---- & $0.0045^{*}$ \\
\hline $\begin{array}{l}\text { Stroke/TIA with oncological } \\
\text { comorbidity and Nattokinase }\end{array}$ & 0.7718 & $0.0045^{*}$ & ---- \\
\hline
\end{tabular}

Table 2. Multiple comparison test in patients with stroke and TIA receiving Nattokinase.

\begin{tabular}{|l|c|c|c|}
\hline & Recurrent Stroke/TIA & Stroke/TIA with Nattokinase & $\begin{array}{c}\text { Stroke/TIA with oncological } \\
\text { comorbidity and Nattokinase }\end{array}$ \\
\hline Mean & 1.3333 & 48 & 8.5 \\
\hline Standard deviation (SD) & 0.8165 & 30.6725 & 4.9295 \\
\hline $\begin{array}{l}\text { Standard error of the mean } \\
\text { (SEM) }\end{array}$ & 0.3333 & 12.522 & 2.0125 \\
\hline $95 \%$ CI of mean & $(0.48)-(2.19)$ & $(15.81)-(80.19)$ & $(3.33)-(13.67)$ \\
\hline
\end{tabular}

Table 3. Intra-group analysis and statistical parameters of the separate categories.

\begin{tabular}{|l|l|l|l|}
\hline & Recurrent Stroke/TIA & $\begin{array}{l}\text { Stroke/TIA with ASA/Clopi- } \\
\text { dogrel }\end{array}$ & $\begin{array}{l}\text { Stroke/TIA with oncologi- } \\
\text { cal comorbidity and ASA/ } \\
\text { Clopidogrel }\end{array}$ \\
\hline Recurrent Stroke/TIA & ---- & $0.0014^{*}$ & 0.9038 \\
\hline $\begin{array}{l}\text { Stroke/TIA with ASA/Clopi- } \\
\text { dogrel }\end{array}$ & $0.0014^{*}$ & ---- & $0.0034^{*}$ \\
\hline $\begin{array}{l}\text { Stroke/TIA with oncologi- } \\
\text { cal comorbidity and ASA/ } \\
\text { Clopidogrel }\end{array}$ & 0.9038 & $0.0034^{*}$ & ----- \\
\hline
\end{tabular}

Table 4. Multiple comparison test in patients with stroke and TIA receiving ASA/Clopidogrel.

\begin{tabular}{|l|c|c|c|}
\hline & Recurrent Stroke/TIA & $\begin{array}{c}\text { Stroke/TIA with ASA/Clopi- } \\
\text { dogrel }\end{array}$ & $\begin{array}{c}\text { Stroke/TIA with oncologi- } \\
\text { cal comorbidity and ASA/ } \\
\text { Clopidogrel }\end{array}$ \\
\hline Mean & 1.5 & 47.5 & 6 \\
\hline Standard deviation (SD) & 1.0488 & 31.2202 & 3.2863 \\
\hline $\begin{array}{l}\text { Standard error of the mean } \\
\text { (SEM) }\end{array}$ & 0.4282 & 12.7456 & 1.3416 \\
\hline $95 \%$ CI of mean & $(0.4)-(2.6)$ & $(14.74)-(80.26)$ & $(2.55)-(9.45)$ \\
\hline
\end{tabular}

Table 5. Intra-group analysis and statistical parameters of the separate categories.

Within the 3 and 6-month monitoring, there were three recurrent ischemic strokes, two TIAs, and cardiovascular complications were observed in three of the subjects.

In the control group with ASA/Clopidogrel intake within 30 days four patients had cerebral infarctions and TIA. Within 3 and 6 months of follow-up there were two cases of TIA, three of stroke and three of cardiovascular complications. The distribution of cardiac and cerebral complications is presented in Figure 2.

When comparing subsequent cerebrovascular accidents, no statistically significant difference was found in the two groups $(\mathrm{p}=0.18$, calc. $\mathrm{t}=1.73$, crit. $\mathrm{t}=3.18$ ). The incidence of cardiovascular problems is identical. When comparing the total number of vascular complications again, no statistically significant difference was found $(\mathrm{p}=0.51$, calc. $\mathrm{t}=0.69$, crit. $\mathrm{t}=2.26$ ).

According to the correlation coefficient $\mathrm{R}$, in the group of patients taking Nattokinase, a positive and upward relationship was observed between the individual values (Figure 3 ). $\mathrm{R}^{2} 100 \%$ is $20 \%$ of the power of influence of the dependent variable, which is an unsignificant result and requires the inclusion of other independent variables $(\mathrm{k} 2=0.5528)$. In the control group, a positive and upward dependence 
can be commented on according to the same coefficient (Figure 4 ). $\mathrm{R}^{2}$ $100 \%$ is only $3.0 \%$ of the power of influence of the dependent variable $(\mathrm{k} 2=0.9697)$ [20-22].

Using linear regression analysis, it was found that the null hypothesis H0 was not rejected (by standard criterion $\mathrm{p}<0.05$ ) or the regression model did not explain the variability in the dependent magnitude of the number of subsequent cerebrovascular events (Figures 3 and 4). Between the separate groups: recurrent stroke/TIA for 1, 3 and 6 months, total number of cerebral infarctions and TIA with Nattokinase and stroke/TIA with oncological comorbidity at a certain value $\mathrm{p}<0.05$ statistical significance was observed: $\mathrm{p}=0.0008$, calc. $\mathrm{F}=11.7711$, crit. $\mathrm{F}=3.6823$. The Tukey HSD multiple comparison test revealed statistical differences between the categories of stroke/TIA and stroke/TIA with Nattokinase, at $\mathrm{p}<0.01$ and between stroke/TIA with Nattokinase and those with oncological comorbidity, $\mathrm{p}<0.01$, HSD [0.05] $=26.96$; HSD [0.01] $=35.8$ (Tables 2 and 3). Comparison among control patients and a certain value $\mathrm{p}<0.05$ a statistical significance was also found: $\mathrm{p}=0.0009$, calc. $\mathrm{F}=11.7327$, crit. $\mathrm{F}=3.6823$. The differences are related to the groups of recurrent cerebrovascular events and a total number of stroke/TIA with ASA/Clopidogrel at $\mathrm{p}<0.01$, and stroke/TIA with ASA/ Clopidogrel and group with concomitant malignant disease, $\mathrm{p}<0.01$, HSD $[0.05]=27.25$; HSD $[0.01]=35.86$ (Tables 4 and 5)

Within a 6-month follow-up period, transient hematuria (1) and cases of gastrointestinal discomfort (2) were noted in patients receiving Nattokinase. In the control group with standard antithrombotic prevention, epistaxis (1), skin changes with the character of ecchymoses (1) and gastrointestinal complaints (2) were reported. The ratio of possible adverse reactions between the two categories is 3.13\%:4.21\%, statistically insignificant $(\mathrm{p}=0.64$, calc. $\mathrm{t}=0.52$, crit. $\mathrm{t}=3.18)$ and confirms the conclusion of the literature and own initial data for a safe profile of the enzyme Nattokinase [23-26].

Despite the serious limitations of the study (a small number of selected patients, short observation period, lack of information about ASA and Clopidogrel resistance in control group participants etc.) there is no statistically reliable difference with the occurrence of vascular (brain and cardiac) complications between the two groups. Patients who take Nataspin $\mathrm{H}^{\otimes}$ had a higher incidence of premorbid oncological conditions. One possible explanation is related to the associated mechanisms of hypercoagulation that have led to changes in blood viscosity and the development of arterial thrombosis - essential factors in the pathogenesis of ischemic disorders of the cerebral circulation [27].

\section{Conclusion}

The concept of Nattokinase as an alternative of standard prevention is interesting, original and promising, in line with the current trends for an interdisciplinary approach and the principles of personalised medicine.

\section{Acknowledgments}

This study was partially supported by a grant from Valentis, Bulgaria, Europe.

\section{References}

1. Milanov Iv, Stamenova P, Casso V. National Consensus for Prevention, Diagnosis and Treatment of Cerebrovascular Diseases. Bulgarian Neurol. 2018; 19: 3-6.

2. Montalvo MJ, Siket MS, Jayaraman MV, et al. (2016) Advances in the management of transient ischaemic attack and stroke. Euro Med J Neuro. 2016; 4: 101-107.

3. Michos ED, Ardehali R, Blumenthal RS, et al. Aspirin and Clopidogrel Resistance. Mayo Clinic Proceedings. 2006; 81: 518-526.

4. Mega JL, Close SL, Wiviott SD, et al. (2009) Cytochrome P-450 polymorphism and response to clopidogrel. N Engl J Med. 2009; 360: 354362 .
5. Nissen SE. Pharmacogenomics and clopidogrel: irrational exuberance? JAMA 2011; 306: 2727-2728.

6. Bhatt DL, Pare G, Eikelboom JW, et al. On behalf of the CHARISMA investigators: The relationship between CYP2C19 polymorphisms and ischaemic and bleeding outcomes in stable outpatients: the CHARISMA genetics study. Eur Heart J. 2012; 33: 2143-2215.

7. Maslarov D, Drenska D, Micova O, et al. Pharmacogenomic Assessment of Clopidogrel - A Step Toward Personalized Medicine For Patients With Ischemic Stroke. XXI World Congress of Neurology. J Neurol Sci. 2013; 333:Q: e262-e263, A-525-0003-02854.

8. Maslarov D. Cerebrovascular Diseases - Recent Evaluations and Ideas for Improving the Public Health. Dissertation for degree "Doctor of Science". Medical University, Sofia. Bulgarian. 2012.

9. Chen H, McGowan EM, Ren N, et al. Nattokinase: A Promising Alternative in Prevention and Treatment of Cardiovascular Diseases. Biomark Insights 2018; 13: 1177271918785130.

10. Ahmed HH, Nevein NF, Karima A, et al. Miracle enzymes serrapeptase and nattokinase mitigate neuroinflammation and apoptosis associated with Alzheimer's disease in experimental model. WJPPS 2013; 3: 876-891.

11. Ji H, Yu L, Liu K, et al. Mechanisms of Nattokinase in protection of cerebral ischemia. Eur J Pharmacol. 2014; 745: 144-151.

12. Sumi $\mathrm{H}$, Hamada $\mathrm{H}$, Tsushima $\mathrm{H}$, et al. A novel fibrinolytic enzyme (nattokinase) in the vegetable cheese Natto; a typical and popular soybean food in the Japanese diet. Experientia. 1987; 43: 1110-11.

13. Urano T, Ihara $\mathrm{H}$, Umemura $\mathrm{K}$, et al. The profibrinolytic enzyme subtilisin NAT purified from Bacillus subtilis cleaves and inactivates plasminogen activator inhibitor type 1. J Biol Chem. 2001; 276: 24690-96.

14. Weng Y, Yao J, Sparks S, et al. Nattokinase: an oral antithrombotic agent for the prevention of cardiovascular disease. Int J Mol Sci. 2017; 18: E523.

15. Jensen GS, Lenninger M, Ero MP, et al. Consumption of nattokinase is associated with reduced blood pressure and von Willebrand factor, a cardiovascular risk marker: results from a randomized, double-blind, placebo-controlled, multicenter North American clinical trial. Integr Blood Press Control. 2016; 9: 95-104.

16. Xie S, Yu Z, Liu X. Preparation of nattokinase and study on its hypolipidemic effect. Chin J Biochem Pharm. 2015; 35: 17-20.

17. Wang JM, Chen HY, Cheng SM, et al. Nattokinase reduces brain infarction, fibrinogen and activated partial thromboplastin time against cerebral ischemia-reperfusion injury. J Food Drug Anal. 2012; 3: 686-91.

18. Ahn YJ, Kim MH, Kim J, et al. Neuroprotective effect of nattokinase mediated by inhibition of platelet aggregation and thrombosis in photothrombotic stroke. Stroke. 2015; 46: A-PW262.

19. Adams Jr HP, Bendixen BH, Kappelle LJ, et al. Classification of subtype of acute ischemic stroke. Definitions for use in a multicenter clinical trial. TOAST. Trial of Org 10172 in Acute Stroke Treatment. Stroke. 1993; 24: 35-41.

20. Fotheringham AS, Wong DWS. The Modifiable Areal Unit Problem in Multivariate Statistical Analysis. Environment Planning A: Economy Space. 1991; 23: 1025-1044.

21. Krousel-Wood MA, Chambers RB, Muntner P. Clinicians' guide to statistics for medical practice and research: part I. Ochsner J. 2006; 6: 68-83.

22. Krousel-Wood MA, Chambers RB, Muntner P. Clinicians' Guide to Statistics for Medical Practice and Research: Part II. Ochsner J. 2007; 7: 3-7.

23. Ren NN, Chen HJ, Li Y, et al. A clinical study on the effect of nattokinase on carotid artery atherosclerosis and hyperlipidaemia. Chinese Med J. 2017; 97: 2038-2042.

24. Petrovsky P. Stroke prevention in patients with atrial fibrillation using the natural product nattokinase as an alternative of conventional anticoagulation. Bulgarian Cardiology XXIII. 2017; 4: 51-55.

25. Simova I, Simov D, Klisurski M, et al. Nattokinase - fibrinolytic, antihypertensive and lipid-lowering potential. An application in Clinical Practice. MD XV. 2018; 1: 87-89.

26. Maslarov D, Drenska D. Nattokinase in Patients with Acute Ischemic Stroke and TIA - preliminary data. XVII National Congress of Neurology with International Participation. Abstract in Bulgarian Neurol. 2018; 19:2:44, A-121.

27. Dardiotis E, Aloizou AM, Markoula S, et al. Cancer-associated stroke: Pathophysiology, detection and management (Review). Int J Oncol. 2019; 54: 779-796. 